\title{
Evaluation of efficacy, safety, pain perception and health-related quality of life of percutaneous ethanol injection as first- line treatment in symptomatic thyroid cysts
}

\author{
Jordi L. Reverter ${ }^{1 *}$, Núria Alonso ${ }^{1}$, Marta Avila², Anna Lucas', Dídac Mauricio and Manel Puig-Domingo ${ }^{1}$
}

\begin{abstract}
Background: To evaluate the efficacy, safety, pain perception and health-related quality of life (QoL) of percutaneous ethanol injection treatment (PEIT) as an alternative to thyroid surgery in symptomatic thyroid cysts.

Methods: Thirty consecutive patients ( $46 \pm 10$ years; $82 \%$ women) with symptomatic benign thyroid cysts relapsed after drainage were included. In all cases, cytology prior to treatment, maximum cyst diameter and volume were determined. PEIT was conducted using the established procedure, and the volume of fluid removed and pain perceived by the patient were assessed. In each procedure, the volume of alcohol instilled was $<2 \mathrm{ml}$. After follow-up, final cyst diameter and volume were determined and the persistence of symptoms and QoL were assessed by a questionnaire (SF-36).

Results: Mean symptom duration was $10 \pm 20$ months. A single session of PEIT was required to complete the procedure in $45 \%$ of patients, two in $31 \%$ and three in $13 \%$. Mean initial maximum cyst diameter was $3.5 \pm 1.0 \mathrm{~cm}$ and mean extracted liquid volume $61 \pm 36 \mathrm{ml}$. During PEIT, $39 \%$ of patients experienced virtually no pain, $43 \%$ mild pain and $17 \%$ moderate pain. No complications of PEIT were observed. After $12.1 \pm 1.4$ months of follow-up, cysts were reduced more than $70 \%$ in volume in $86.3 \%$ of patients, more than $80 \%$ in $61.9 \%$ and more than $90 \%$ in $42 \%$. On the health-related QoL SF-36 questionnaire, patient scores 6 months post-PEIT did not differ significantly from those of the healthy Spanish population. With respect to cosmetic complaints or local symptoms of compression, PEIT-treated patients presented an initial score of $22 \pm 8$ and $13 \pm 5$ after treatment $(p<0.05)$.
\end{abstract}

Conclusions: In our experience, percutaneous ethanol injection has prove to be an effective, safe and well-tolerated firstline treatment of symptomatic thyroid cysts.

Keywords: Thyroid cysts, Percutaneous ethanol injection, Health-related quality of life, Pain

\section{Background}

Thyroid nodules are common in the general population with a prevalence of palpable lesions between 3 and $10 \%$ [1]. Moreover, non-palpable ultrasound-detected thyroid nodules are present in 20 to $60 \%$ of healthy individuals [2]. Thyroid cysts are characterised by their liquid contents and are mostly benign [3]. After percutaneous drainage, most

\footnotetext{
* Correspondence: reverter.germanstrias@gencat.cat

'Department of Endocrinology and Nutrition, Germans Trias i Pujol Health Science Research Institute and Hospital, CIBER of Diabetes and Associated Metabolic Diseases (CIBERDEM), Universitat Autònoma de Barcelona, Carretera de Canyet s/n, 08916 Badalona, Spain

Full list of author information is available at the end of the article
}

cystic lesions (around $80 \%$ ) refill and enlarge over time. In cases of recurrent thyroid cysts producing aesthetic complaints or compressive symptoms, surgery has been the first-line treatment to date [4]. Since the late 1990s, sonography-guided percutaneous ethanol injection treatment (PEIT) has emerged as a safe and effective conservative alternative to surgical excision [5]. The injection of 95-99 \% ethanol into the cyst cavity induces thrombosis of small vessels and coagulative necrosis surrounded by interstitial oedema and granulomatous inflammation, followed by fibrosis, shrinkage and reduction in the volume of the lesion [5, 6]. A randomised study from Bennedbaek and 
Hegedüs reported a significant $82 \%$ volume reduction in the PEIT group $(n=33)$ compared to $18 \%$ in the saline treated group $(n=33)$ [7], with few complications, thereby providing data permitting this technique to be considered as a reliable alternative to thyroid surgery. In this respect, recent guidelines in the USA and Europe [2] state that PEIT is a clinically-effective, non-surgical option for repeatedly drained recurrent thyroid cysts. However, there is a paucity of reports in the literature from few centres describing the outcomes of this technique for avoiding surgery in these patients when it is introduced in systematic clinical practice.

Furthermore, as a recent review of the Cochrane Collaboration established, previous studies on this technique did not provide information on health-related quality of life (QoL) and the authors of the review thus suggested that this important measure for the patient should also be a primary end-point in future trials. [8].

Herein, we reported our results on effectiveness, safety, pain perception and health-related QoL in a group of patients with symptomatic recurrent thyroid cysts after PEIT.

\section{Methods}

Thirty consecutive patients treated at the outpatient clinic of the Endocrinology and Nutrition Department of the Germans Trias i Pujol Universtity Hospital, Badalona, in 2013 and referred for evaluation of a cervical nodule or nodular goiter were included. A case was considered for PEIT if it met all the following selection criteria: 1 ) age $>18$ years; 2 ) normal thyroid function tests; 3) no major comorbidities; 4) no history of neck irradiation; 5) cystic or predominantly cystic (>80 \% cystic component) thyroid nodule; 6 ) compressive symptoms or aesthetic complaints; and 7) voluntary patient decision to undergo PEIT instead of open surgery after careful explanation of the procedure. The study was conducted in accordance with the Declaration of Helsinki and was approved by the local Human Research Ethics Committee of the Hospital Germans Trias i Pujol. All participants gave their written informed consent.
In all cases, a medical history was taken and physical examination performed and blood samples were obtained for analytical determinations. Serum free thyroxine (fT4) and TSH were measured by electrochemiluminescence immunoassay $\left(\right.$ Siemens $^{\odot}$, Los Angeles, CA, USA).

\section{Ultrasound examination}

An echography examination was performed in each patient by an experienced operator (JLR) using a 12$15 \mathrm{MHz}$ linear transducer device. Morphological evaluation included description of the thyroid echostructure and measurement of the diameters and ultrasound characteristics of each detected nodule (Fig. 1, panel a). Volumetric assessment of the nodules was based on the use of an ellipsoid model [9]. With this rotating ellipsoid model, the height, width and depth of each nodule were measured and multiplied. The obtained result was then multiplied by the mathematical constant or correction factor 0.524 [10]. Fine-needle aspiration (FNA) cytology was performed in all cases to obtain samples from all nodules $>1.0 \mathrm{~cm}$ in diameter and those between 0.5 and $1.0 \mathrm{~cm}$ if they presented suspicious ultrasound characteristics according to the American Society of Ultrasonography [11] such as the presence of microcalcifications, irregular borders, increased central flow on Doppler examination, taller than wide diameter, hypoechogenicity and absence of halo. In the case of cysts, FNA was performed from the capsule or solid part of the nodule and for drainage of the cyst contents, and cytological analysis was performed. Patients were offered PEIT if the cyst presented significant regrowth after the first drainage with compressive symptoms or aesthetic complaints and cytological analysis was benign and preferred not to undergo thyroid surgery after extensive explanation of the procedure.

\section{Percutaneous ethanol injection treatment procedure}

Positions of the patient and operator were similar to those of the FNA procedure, with the patient lying with their neck in hyperextension. After skin sterilisation, and
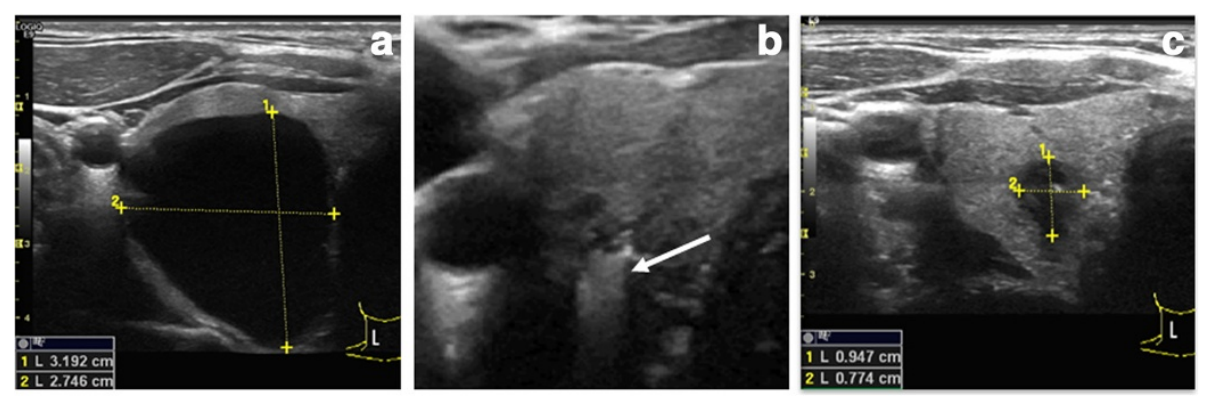

Fig. 1 Transverse ultrasound scans of one cyst before (panel a), during (panel b) and after (panel c) percutaneous ethanol injection treatment (PEIT). During PEIT, cystic lumen was filled with instilled ethanol (arrow). Panel c shows marked decrease in size 
under ultrasound guidance, a $21 \mathrm{G}$ needle mounted on a connector with a line extension and a $20 \mathrm{ml}$ syringe were used to empty the contents of the cyst. Subsequently, $0.5 \mathrm{ml}$ of $5 \%$ lidocaine was infused into the cyst cavity and, after a 2 min wait, $99 \%$ ethanol was injected with a slow movement of the needle to reach the major part of the inner face of the cyst capsule (Figure 1, panel b). The patient was instructed to signal any feeling of pain to prevent the spread of alcohol into the neck structures. The amount of ethanol injected was $50 \%$ of the volume of liquid extracted with a maximum of $2 \mathrm{ml}$. The needle tip was constantly visualsed during the procedure. In no case were more than $2 \mathrm{ml}$ injected. The ethanol was not re-extracted and patients were discharged after a 20-30minute observation period.

\section{Follow-up}

Patients were followed up weekly for 1 month, monthly for 3 months and every 3 months thereafter. Ultrasound examination was performed at each appointment to evaluate the characteristics and volume of the residual lesion (Fig. 1, panel c).

In cases of symptomatic cyst regrowth confirmed by echography, a new PEIT was performed with the same protocol followed by weekly appointments. This procedure was repeated until permanent and significant cyst reduction was achieved or the patient decided not to repeat the PEIT procedure. No limits were set in the number of PEIT procedures.

\section{Response parameters}

In each patient, the percentage of volume reduction was calculated by the formula [(V1-V2)/V1] $\times 100$ in which $\mathrm{V} 1$ is the initial cyst volume and V2 the final cyst volume.

Patients were requested to grade the sensation of pain suffered with the alcohol injection immediately after the procedure, using a visual scale of $10 \mathrm{~cm}$ between the absence of pain (0) and excruciating pain (10) in which each $1 / 3$ of the scale corresponded, from the lowest to the highest, to the following categories: mild pain, moderate pain and severe pain.

\section{Health-related quality of life}

The Spanish version of the SF-36 questionnaire provided online by www.sf-36.org/ was administered to each patient 6 months after the last PEIT. The SF-36 questionnaire is a multipurpose, short-form health survey, commonly used to evaluate patients' health-related QoL in clinical practice. Eight domains are assessed in this questionnaire, including physical functioning, role limitation due to physical problems, bodily pain, general health, vitality, social functioning, role limitation due to emotional problem, and mental health. The eight health domains were used to provide a physical component summary (PCS) and a mental component summary score (MCS). Interpretation of the SF-36 Health Survey was greatly simplified with the norm-based scoring (NBS) of its health domain scales and component summary measures. In NBS, each scale is scored to have the same average (50) and the same standard deviation [10], signifying that each point equals one-tenth of a standard deviation [12]. For all scales and summary measures mean group scores below 47 can be interpreted as being below the average range for the general population.

With a view to assessing the specific symptoms of neck cysts derived, we created a domestic non-validated questionnaire including ten items related to the most frequent goitre symptoms described in the literature. These symptoms were related to visible enlargement of the neck (cosmetic complaint), feeling of pressure in the neck, throat pressure, sore throat, pain in the throat radiating to the ears, difficulty in swallowing, throat clearing often, shortness of breath and hoarseness. Each item was scored between 1 (none of the time), 2 (a little of the time), 3 (some of the time), 4 (most of the time) and 5 (all of the time), and the final score was the sum of the ten items. Thus, a score could range from ten (absence of symptoms) to 50 (patient's worst feeling of symptoms). Compressive symptoms on the trachea were evaluated by chest X-ray.

\section{Statistical analysis}

Continuous variables were expressed as mean $\pm \mathrm{SD}$ or median (interquartile range) and categorical variables as percentages. Student's t-test was used for comparisons between continuous variables and Pearson's correlation test for correlation analyses. A $p$ value $<0.05$ was considered statistically significant and correlations were considered significant for Pearson's correlation coefficient if $\geq 0.25$. For comparison of categorical variables, the chisquare test or Fisher's exact test were used where appropriate. Data analyses were made using the Statistical Package for Social Sciences (SPSS $^{\odot}$, Chicago, IL, USA) for Windows ${ }^{\oplus}$, Version 15.0.

\section{Results and discussion}

Thirty subjects with symptomatic thyroid cysts $(46 \pm$ 10 years; $82 \%$ women) were included in the protocol. Mean duration of symptoms was 10 (1-36) months and, in all cases, TSH and fT4 were within the normal range. FNA cytology was benign in all samples obtained (haemorrhagic-cystic lesion, colloid cyst or non-malignant lesion) from the predominantly cystic nodules and the other nodules with sonographic criteria for FNA cytology [13]. Mean maximum cyst diameter before drainage was $3.5 \pm 1.0 \mathrm{~cm}$ with a calculated initial volume of $18.2 \pm 15.5 \mathrm{ml}$ (median $14.5 \mathrm{ml}$ ). Mean total volume 
extracted from the cysts in all procedures performed was $61.0 \pm 36.5 \mathrm{ml}(2-265 \mathrm{ml})$.

The required number of PEIT for permanent reduction in cysts and a symptom-free situation was one in $45.5 \%$ of cases, two in $31.8 \%$ and three in $13.6 \%$. Two patients with the largest cysts in this series (total volume extracted: 145 and $265 \mathrm{ml}$ ) were treated four and six times, respectively. Initial calculated cyst volume was significantly greater in the group of patients who required three or more PEIT procedures compared with those treated with one or two PEITs $(29.6 \pm 25.5 \mathrm{ml} v s$. $15.5 \pm 10.6 \mathrm{ml}$, respectively; $p<0.05$ ).

In all cases requiring more than one treatment, cyst recurrence was observed during the first month posttreatment, both for the first PEIT and subsequent procedures. No delayed recurrences were observed.

The sensation of pain reported during PEIT was virtually absent (39.1\%), mild (43.5\%) or moderate (17.4\%). In all cases, this was a transient sensation of pain with relief within minutes. No patient felt intense pain and none suffered other complications.

After 12.1 \pm 1.4 months of follow-up mean calculated cyst volume reduction was $85.9 \pm 14.7 \%$. In all cases, cyst volume was reduced more than $50 \%$ from the basal. Cysts were reduced more than $70 \%$ in volume in $86.3 \%$ of patients, more than $80 \%$ in $61.9 \%$ and more than $90 \%$ in $42 \%$. Final mean maximum thyroid cysts diameter was $1.3 \pm 0.6 \mathrm{~cm}$. Total disappearance of the cysts was observed in three cases.

Regarding the characteristics of cystic lesions, 19 of the cysts were purely cystic nodules, and 11 mixed or predominantly cystic nodules (with a $<20$ solid component). No differences were observed between cystic and mixed nodules in calculated baseline volume (19.7 \pm 16.1 vs $14.8 \pm 8.7 \mathrm{ml}$, respectively; $p=0.3$ ), calculated final volume $(1.6 \pm 2.6$ vs. $1.9 \pm 1.6 \mathrm{ml}$, respectively; $p=0.7)$ and volume reduction $(88.2 \pm 12$ vs. $80.3 \pm 17.5 \%$ respectively; $p=0.2$ ). The number of PEIT sessions required was also similar (one in 47.3 vs. $50.0 \%, p=0.8$; in purely cystic and mixed cysts, respectively).

Table 1 summarizes the final volume observed and the volume reduction according to the calculated initial volume of the cysts. No significant differences were observed between cysts $>15 \mathrm{ml}$ in the calculated final volume $(p=0.7)$.

Table 1 Cyst volume results

\begin{tabular}{lccc}
\hline & $<15 \mathrm{ml}(n=15)$ & $>15 \mathrm{ml}(n=15)$ & $P$ value \\
\hline Baseline volume $(\mathrm{ml})$ & $8.4 \pm 4.1$ & $28.8 \pm 13.1$ & 0.001 \\
Final volume $(\mathrm{ml})$ & $1.6 \pm 1.5$ & $1.9 \pm 2.9$ & 0.7 \\
Volume reduction $(\%)$ & $78.1 \pm 15.8$ & $93.7 \pm 7.5$ & 0.002 \\
\hline
\end{tabular}

Results on calculated cyst volume and percentage of volume reduction in patients treated with percutaneous ethanol injection treatment (PEIT) according to their initial volume
As expected, volume reduction was significantly $(p=0.002)$ greater in nodules $>15 \mathrm{ml}(78.1 \pm 15.8 \%)$ compared to those $<15 \mathrm{ml}(93.7 \pm 7.5 \%), p=0.002$.

In the health-related QoL SF-36 questionnaire, normbased PCS [52.4 (4.4)] and MCS [49.3 (4.8)] scores of the patients 6 months after PEIT did not significantly differ from those of the healthy Spanish population [14].

With respect to specific questions addressing to cosmetic complaints or local symptoms of compression, PEIT-treated patients presented an initial score of $22 \pm 8$ and after treatment $13 \pm 5(p<0.05)$ with $47 \%$ of cases reporting a score of ten, i.e. absence of symptoms.

In the correlation analysis, a significant relationship observed were between calculated initial cyst volume and evolution time $(r=-0.4, p=0.02)$ and between baseline calculated volume and volume reduction $(r=-0.1$, $p=0.04$ ).

\section{Discussion}

In our experience, percutaneous ethanol treatment is a safe, well-tolerated and effective procedure for the treatment of symptomatic benign thyroid cysts, achieving relief or the disappearance of the aesthetical complaints and/or compressive symptoms in all treated patients. Moreover, patients who underwent this technique showed a healthrelated quality of life related similar that of the normal population according to a validated questionnaire.

Since the introduction of PEIT for thyroid cysts in clinical practice, some groups from Italy [15-18], Denmark [7] and South Korea [19, 20] reported a significant high success rate (more than $80 \%$ ) with no significant side effects. Based on these results, the major endocrine society guidelines state that treatment of recurrent benign thyroid cysts with ethanol in experienced hands is a clinically effective, non-surgical option for purely or predominantly cystic thyroid nodules that recur after repeat aspirations $[2,21]$. The results obtained in our group of treated patients after the PEIT procedure was introduced as a regular practice at our hospital concur with those good results report in terms of efficacy and safety, with more than $70 \%$ volume reduction in more than $85 \%$ of patients and the disappearance of aesthetic complaints or compression in almost half of cases and significant relief in the rest.

In our protocol, we offered PEIT after the first cyst drainage. With this approach, the benign nature of the lesion was confirmed by cytological examination of the samples and the refilling of cysts that occurred in all cases in this series. The number of PEIT sessions required ranged from one to six depending on initial cyst size and volume. However, the majority of cases $(87.3 \%$ in our series) were resolved with the first or second procedure and whether more than two treatments were required depended on initial cyst volume. In our opinion 
this feature is important in order to discuss with the patient before the procedure the eventual needing of more than one or two PEITs.

The volume of ethanol used in PEIT is a matter of debate. The reported amounts of ethanol administered ranged from 35 to $100 \mathrm{~mL}$ depending on the authors $[6,7,16,19]$, with a recommended maximum single infusion of $10 \mathrm{~mL}$, although treatments with the instillation of up to $30 \mathrm{~mL}$ have been reported [22]. In our protocol, we decided to use a maximum ethanol volume of less than $2 \mathrm{~mL}$ in all cases. We, like others [23], believe this cautious approach offers several advantages such as less pain, and lower risk of ethanol leakage into surrounding tissues and, owing to collapse of the cyst after drainage of its liquid content, this small amount of ethanol is sufficient to contact with the wall without dilution. On the other hand, we decided not to re-aspirate the ethanol after it was instilled into the empty cyst cavity. Previous studies reported similar results in the non-re-aspiration and re-aspiration groups [22]; however, the non-re-aspiration technique is less time consuming and reduces the number of injections in each patient, and minimizes the possibility of the needle protruding from the cyst cavity due to longer handling during the reaspiration phase of the procedure. The efficacy of the procedure in reducing of cyst volume and symptoms in the present study supports this approach.

A major concern in all interventional procedures is patient comfort. We specifically tested pain sensation during PEIT using a visual scale, and it is noteworthy that over $80 \%$ of patients reported virtually absent or mild pain and none of the treated group subjects described it as greater than moderate. Previous reports described a transient mild or burning sensation, which was more intense in the largest cysts [7, 24]. Based on these results no local anaesthesia is usually recommended. In our protocol, the injection of a small amount of lidocaine into the cyst cavity before ethanol instillation was included. The high rate of patients reporting virtually no pain (almost $40 \%$ ) supports continuing with this procedure.

There is a lack of information in the literature regarding the quality of life of patients with recurrent thyroid cysts treated with ethanol [8]. We addressed this question using a general health questionnaire (SF 36) and a specific questionnaire for symptoms of goitre, we detected normal health quality status as reflected in the SF-36 results and a significant improvement in goitre symptoms using the specific questionnaire. We tested general health only after performing PEIT since, in thyroid nodules with no associated hypo- or hyperthyroidism, the main complaint is local neck symptoms. Post-treatment evaluation confirmed that the procedure did not affect the patient well-being, which therefore had to be similar to that of the general population in terms of SF-36 score. A summary of physical and mental scores showed good status in these patients compared the normal population. Moreover, the specific symptoms of goitre improved dramatically until its disappearance in many patients. To our knowledge, no previous studies had been conducted on QoL in patients with ethanol-treated thyroid cysts and our results add new data in favour of PEIT as an alternative to thyroid surgery. The American and European guidelines $[2,21]$ recommend PEIT for thyroid cysts, but only those that recur after repeated aspirations. Based on our results and previous reports $[6,7,16,19,22,23]$, we believe PEIT should be included as the treatment of choice after the first unsuccessful drainage of cysts. Although the protocol for PEIT is not universally unified regarding the amount of alcohol instilled, re-aspiration of the instilled ethanol and the different use of anaesthesia according to groups, the general results obtained by PEIT are not affected by these protocol differences. Thus, albeit not mandatory, it would be convenient to have a homogeneous protocol established by scientific societies and ad hoc guidelines based on the reported results and local experience $[25,26]$.

\section{Conclusions}

PEIT in recurrent thyroid cysts appears to be a highly efficacious and safe technique. The improvement in clinical conditions obtained with very low pain sensation, absence of complications and good QoL perceived after the procedure render PEIT an optimum and more recommendable alternative to thyroid surgery. In our opinion, future guidelines should include PEIT prior to surgical treatment and establish a uniform consensus protocol.

\section{Competing interests}

The authors declare that they have no competing interests.

\section{Authors' contributions}

$J \mathrm{R}$, carried out the ultrasound-guided percutaneous ethanol treatment and coordinated the study. NA, AL, MPD, DM participated in the patient's selection and follow-up. MA participated in pathological analysis. JLR, NA, MPD, DM conceived of the study, and participated in its design and helped to draft the manuscript. All authors read and approved the final manuscript.

\section{Acknowledgements}

The authors acknowledge to Isabel Ordoñez, Maribel Cuadrado and Lucia Cano their contribution to this work. We also thank Christine O'Hara for valuable help with the English version of the manuscript. CIBER of Diabetes and Associated Metabolic Diseases (CIBERDEM) is an initiative from Instituto de Salud Carlos III.

\section{Author details}

${ }^{1}$ Department of Endocrinology and Nutrition, Germans Trias i Pujol Health Science Research Institute and Hospital, CIBER of Diabetes and Associated Metabolic Diseases (CIBERDEM), Universitat Autònoma de Barcelona, Carretera de Canyet s/n, 08916 Badalona, Spain. ²Department of Pathology, Germans Trias i Pujol Health Science Research Institute and Hospital, Universitat Autònoma de Barcelona, Badalona, Spain. 
Received: 16 April 2015 Accepted: 16 November 2015 Published online: 26 November 2015

\section{References}

1. Bryan R Haugen MD, Erik K. Alexander, Keith C. Bible, Gerard Doherty, Susan J Mandel, Yuri E. Nikiforov, Furio Pacini, Gregory Randolph, Anna Sawka, Martin Schlumberger, Kathryn G Schuff, Steven I. Sherman, Julie Ann Sosa, D Steward, R Michael Tuttle MD, Leonard Wartofsky: 2015 American Thyroid Association Management Guidelines for Adult Patients with Thyroid Nodules and Differentiated Thyroid Cancer. Thyroid 2015, 14: Epub ahead of print.

2. Gharib H, Papini E, Paschke R, Duick DS, Valcavi R, Hegedüs L, et al. AACE/ AME/ETA Task Force on Thyroid Nodules. American Association of Clinical Endocrinologists, Associazione Medici Endocrinologi, and European Thyroid Association medical guidelines for clinical practice for the diagnosis and management of thyroid nodules. Endocr Pract. 2010;16:1-43.

3. Popoveniuc G, Jonklaas J. Thyroid nodules. Med Clin North Am. 2012;96:329-49.

4. Papini E, Pacella CM, Hegedus L. Diagnosis of endocrine disease: thyroid ultrasound (US) and US-assisted procedures: from the shadows into an array of applications. Eur J Endocrinol. 2014;170:R133-46.

5. Zingrillo M, Torlontano M, Chiarella R, Ghiggi MR, Nirchio V, Bisceglia M, et al. Percutaneous ethanol injection may be a definitive treatment for symptomatic thyroid cystic nodules not treatable by surgery: five-year follow-up study. Thyroid. 1999;9:763-7.

6. Cho YS, Lee HK, Ahn IM, Lim SM, Kim DH, Choi CG, et al. Sonographically guided ethanol sclerotherapy for benign thyroid cysts: results in 22 patients. AJR Am J Roentgenol. 2000;174:213-6.

7. Bennedbaek FN, Hegedüs L. Treatment of recurrent thyroid cysts with ethanol: a randomized double-blind controlled trial. J Clin Endocrinol Metab. 2003;88:5773-7.

8. Bandeira-Echtler E, Bergerhoff K, Richter B. Levothyroxine or minimally invasive therapies for benign thyroid nodules. Cochrane Database Syst Rev. 2014;6:CD004098.

9. Brunn J, Block U, Ruf G, Bos I, Kunze WP, Scriba PC. Volumetric analysis of thyroid lobes by realtime ultrasound [in German]. Dtsch Med Wochenschr. 1981;106:1338-40.

10. Shabana W, Peeters E, De Maeseneer M. Measuring thyroid gland volume: should we change the correction factor? AJR Am J Roentgenol. 2006;186:234-6.

11. Kim EK, Park CS, Chung WY, Oh KK, Kim DI, Lee JT, et al. New sonographic criteria for recommending fine-needle aspiration biopsy of nonpalpable solid nodules of the thyroid. AJR Am J Roentgenol. 2002;178:687-91.

12. Ware JE, Kosinski MA, Keller SD. SF-36 physical and mental health summary scales. A user's manual. Boston: The Health Institute. New England Medical Center; 1994.

13. Muratli A, Erdogan N, Sevim S, Unal I, Akyuz S. Diagnostic efficacy and importance of fine-needle aspiration cytology of thyroid nodules. J Cytol. 2014;31:73-8.

14. Alonso J, Regidor E, Gregorio B, Prieto L, Rodríguez C, De la Fuente L. Valores poblacionales de referencia de la version española del Cuestionario de Salud SF-36. Med Clin (Barc). 1998;111:410-6.

15. Guglielmi R, Pacella CM, Bianchini A, Bizzarri G, Rinaldi R, Graziano FM, et al. Percutaneous ethanol injection treatment in benign thyroid lesions: role and efficacy. Thyroid. 2004;14:125-31.

16. Valcavi R, Frasoldati A. Ultrasound-guided percutaneous ethanol injection therapy in thyroid cystic nodules. Endocr Pract. 2004;10:269-75.

17. Monzani F, Lippi F, Goletti O, Del Guerra P, Caraccio N, Lippolis PV, et al. Percutaneous aspiration and ethanol sclerotherapy for thyroid cysts. J Clin Endocrinol Metab. 1994;78:800-2.

18. Lippi F, Ferrari C, Manetti L, Rago T, Santini F, Monzani F, et al. Treatment of solitary autonomous thyroid nodules by percutaneous ethanol injection: results of an Italian multicenter study. The Multicenter Study Group. J Clin Endocrinol Metab. 1996;81:3261-4.

19. Sung JY, Baek JH, Kim KS, Lee D, Yoo H, Kim JK, et al. Single-session treatment of benign cystic thyroid nodules with ethanol versus radiofrequency ablation: a prospective randomized study. Radiology. 2013;269:293-300.

20. Gharib H, Hegedüs L, Pacella CM, Baek JH, Papini E. Clinical review: Nonsurgical, image-guided, minimally invasive therapy for thyroid nodules. J Clin Endocrinol Metab. 2013;98:3949-57

21. Paschke R, Hegedus L, Alexander E, Valcavi R, Papini E, Gharib H. Thyroid nodule guidelines: agreement, disagreement and need for future research. Nat Rev Endocrinol. 2011;7:354-61.
22. Kim DW, Rho MH, Kim HJ, Kwon JS, Sung YS, Lee SW. Percutaneous ethanol injection for benign cystic thyroid nodules: is aspiration of ethanol-mixed fluid advantageous? Am J Neuroradiol. 2005;26:2111-27.

23. - Halenka M, Karasek D, Frysak Z: Ultrasound-guided percutaneous ethanol injection of small and medium-sized thyroid cysts with relatively small amounts of ethanol. Biomed Pap Med Fac Univ Palacky Olomouc Czech Repub 2014, 20: doi: 10.5507/bp.2014.009 [Epub ahead of print].

24. Del Prete S, Caraglia M, Russo D, Vitale G, Giuberti G, Marra M, et al. Percutaneous ethanol injection efficacy in the treatment of large symptomatic thyroid cystic nodules: ten-year follow-up of a large series. Thyroid. 2002;12:815-21.

25. Fernández-García JC, Mancha-Doblas I, Tinahones FJ. Diagnostic and functional structure of a high-resolution thyroid nodule clinic: An efficiency model. Endocrinol Nutr. 2014;61:553-4.

26. Argüelles I, Tofé S. Importance of ultrasound in a department of endocrinology. Endocrinol Nutr. 2013;60:51-2.

\section{Submit your next manuscript to BioMed Central and we will help you at every step:}

- We accept pre-submission inquiries

- Our selector tool helps you to find the most relevant journal

- We provide round the clock customer support

- Convenient online submission

- Thorough peer review

- Inclusion in PubMed and all major indexing services

- Maximum visibility for your research

Submit your manuscript at www.biomedcentral.com/submit
C) BioMed Central 